\title{
$\gamma$-ray production via IC scattering of the infrared excess from the Be-type star in the binary system PSR B1259-63/SS2883
}

\author{
Brian van Soelen ${ }^{1}$ and Pieter J. Meintjes ${ }^{2}$ \\ Department of Physics, University of the Free State, Bloemfontein, Republic of South Africa \\ ${ }^{1}$ email: vansoelenb@ufs.ac .za ${ }^{2}$ email: MeintjPJ@ufs.ac.za
}

\begin{abstract}
Un-pulsed $\gamma$-ray emission has been detected close to periastron in the pulsar/Be-star binary system PSR B1259-63/SS 2883, believed to originate from the shock front that forms between the stellar and pulsar winds. A likely source of $\gamma$-ray production is the inverse Compton up-scattering of target photons from the Be star by relativistic electrons/positrons in the pulsar wind. In this study the influence of the infrared radiation, emanating from the circumstellar disc, on isotropic inverse Compton $\gamma$-ray production is investigated. It is shown that the scattering of infrared disc photons can increase the $\gamma$-ray flux by a factor $\sim 2$ in the $1-10 \mathrm{GeV}$ range.
\end{abstract}

Keywords. stars: emission-line, Be, binaries: general, gamma rays: theory

\section{Introduction}

The Be X-ray Pulsar binary (Be-XPB) PSR B1259-63/SS 2883 consists of a $48 \mathrm{~ms}$ pulsar in orbit around a Be star (Johnston et al. 1992). The orbit is eccentric $(e \approx 0.87)$ and un-pulsed $\mathrm{TeV} \gamma$-ray emission has been detected around periastron (Aharonian et al. 2005). The system is powered by the spin-down luminosity of the pulsar and the unpulsed radiation is believed to originate from a stand-off shock-front that forms between the pulsar and stellar wind. A likely production mechanism for the $\gamma$-rays is the inverse Compton (IC) scattering of target photons from the Be star (Tavani \& Arons 1997). Previous models of IC scattering in PSR B1259-63/SS 2883 have not considered how the infrared (IR) excess from the circumstellar disc will influence the $\gamma$-ray production.

\section{Modelling}

\subsection{Curve of Growth Method}

The Curve of growth method (as outlined by Waters 1986) was used to model the IR excess associated with SS 2883. Under this model it is assumed that the Be star's circumstellar disc has a half-opening angle $\theta=5^{\circ}$, extends to a radius $R_{\text {disc }}=50 R_{\text {star }}$, and follows a power-law density profile $\rho \propto\left(r / R_{\text {star }}\right)^{-n}$. A Kurucz atmosphere (Kurucz 1979) was fitted to the optical data ( $U B V$ from Westerlund \& Garnier 1989), giving a temperature of $T_{\text {star }}=25000 \mathrm{~K}$, while the IR excess was fitted to 2MASS (Skrutskie et al. 2006) and $M S X$ data (Price et al 2001) assuming $T_{\text {disc }}=0.5 T_{\text {star }}$.

\subsection{Inverse Compton Scattering}

As a first approximation of the effect of the IR excess on IC scattering the total number of scatters is calculated by integrating over the exact isotropic scattering rate equation given in Blumenthal \& Gould (1970). The scattering rate is proportional to the photon distribution, $n(\epsilon)$, which is calculated by using the curve of growth method instead of a 


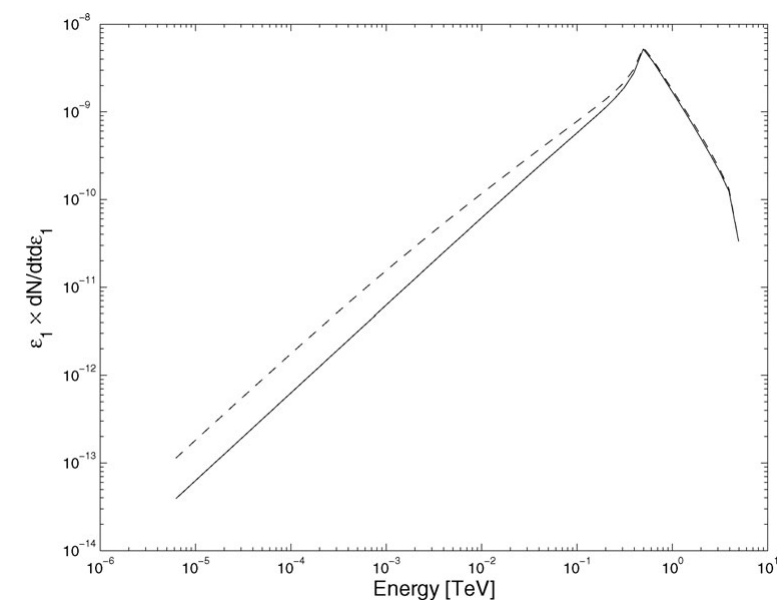

Figure 1. The predicted IC $\gamma$-ray flux in arbitrary unites for PSR B 1295-63/SS 2883. Dashed line includes the flux from the star and disc, while the solid line excludes the disc.

blackbody or mono-energetic photon distribution as was used in previous models. Fig. 1 shows the results of the IC scattering when only the flux from the star (solid line) and when the flux from the star plus the disc (dashed line) is considered, assuming a isotropic electron distribution, with a power index $p=2.2$ between $\gamma=10^{6}-10^{7}$. The modelled flux shows an increase of a factor $\sim 2$ in the $1-10 \mathrm{GeV}$ range.

\section{Conclusion}

The predicted increase in the $\gamma$-ray flux is important for observations of $\gamma$-ray Be-XPB systems, especially given the observational range of Fermi. Future work will consider the anisotropic effects associated with a finite size star and disc.

\section{Acknowledgements}

This publication makes use of data from 2MASS, a joint project of the University of Massachusetts and the Infrared Processing and Analysis Center/California Institute of Technology, funded by NASA and the NSF. This research made use of data from $M S X$; processing of the data was funded by the Ballistic Missile Defense Organization with additional support from the NASA Ofce of Space Science. This research has made use of the NASA/IPAC Infrared Science Archive, operated by the Jet Propulsion Laboratory, California Institute of Technology, under contract with NASA.

\section{References}

Aharonian, F., Akhperjanian, A. G., Aye, K.-M., Bazer-Bachi, A. R. et al. 2005, A\& A, 442, 1 Blumenthal, G. R. \& Gould, R. J. 1970, Reviews of Modern Physics, 42, 237

Johnston, S., Manchester, R. N., Lyne, A. G., Bailes, M. et al. 1992, ApJ (Letters), 387, L37 Kurucz, R. L. 1979, ApJS, 40, 1

Price, S. D., Egan, M. P., Carey, S. J., Mizuno, D. R. et al. 2001, AJ, 121, 2819

Skrutskie, M. F., Cutri, R. M., Stiening, R., Weinberg, M. D. et al. 2006, AJ, 131, 1163

Tavani, M. \& Arons, J. 1997, ApJ, 477, 439

Waters, L. B. F. M. 1986, A\&A, 162, 121

Westerlund, B. E. \& Garnier, R. 1989, A\&AS, 78, 203 